\title{
ANÁLISE TÉCNICO-ECONÔMICA DA PRODUÇÃO DE GASÓLEO MARINHO SINTÉTICO A PARTIR DE ROADMAP
}

\author{
Pedro Ribeiro Siqueira Santos \\ SENAI CETIQT - Centro de Tecnologia da Indústria Química e Têxtil \\ Rua Magalhães Castro, 174 - Riachuelo, Rio de Janeiro - RJ, 20961-020 \\ ribpedross@gmail.com \\ Rinaldo Farias da Luz \\ SENAI CETIQT - Centro de Tecnologia da Indústria Química e Têxtil \\ Rua Magalhães Castro, 174 - Riachuelo, Rio de Janeiro - RJ, 20961-020 \\ RLuz@cetiqt.senai.br \\ Luiz Carlos Fonte Nova de Assumpção \\ SENAI CETIQT - Centro de Tecnologia da Indústria Química e Têxtil \\ Rua Magalhães Castro, 174 - Riachuelo, Rio de Janeiro - RJ, 20961-020 \\ LCAssumpcao@cetiqt.senai.br
}

\begin{abstract}
RESUMO
As emissões provenientes da combustão do gasóleo marinho (MGO) vêm recebendo grande atenção nos últimos anos. A partir disto, novas estratégias são necessárias para produzi-lo com menor teor de contaminantes. De modo a contribuir para essa discussão, o presente trabalho visa estudar a produção deste combustível de maneira sintética (via processo Fischer-Tropsch) a partir de prospecção tecnológica. Para isto, utilizou-se o software Aspen Hysys V10 com o pacote termodinâmico SRK para simular o processo, bem como suas condições operacionais e o Aspen Process Economic Analyzer (APEA) para custear e dimensionar os equipamentos. $\mathrm{O}$ estudo evidenciou que, apesar de apresentar elevados custos operacionais, o processo Fischer-Tropsch é capaz de produzir um MGO dentro das especificações necessárias e com preço competitivo em relação aos convencionais (fósseis) disponíveis no mercado.
\end{abstract}

Palavra-chave: Gasóleo marinho sintético; Fischer-Tropsch; Análise técnico-econômica; Aspen Hysys; Roadmap.

\section{ABSTRACT}

The emissions from marine gas oil (MGO) combustion has been receiving great attention nowadays. From this, new strategies are needed to produce them with lower contaminant content. To contribute to this discussion, the present work aims to study the production of this fuel synthetically (for Fischer-Tropsch process) from technological prospecting. For this, Aspen Hysys V10 with SRK thermodynamic package was used to simulate the process, as well as its operating conditions and Aspen Process Economic Analyzer (APEA) to costing and size the equipment. The study highlighted that, despite having high operating costs, the Fischer-Tropsch process is capable of producing an MGO within the required specifications and competitively priced compared to conventional available on the market.

Keywords: Synthetic marine diesel; Fischer-Tropsch; Technical and economic analysis; Aspen Hysys; Roadmap. 


\section{Como Citar:}

SANTOS, Pedro Ribeiro S.; LUZ, Rinaldo Farias.; ASSUMPÇÃO, Luiz Carlos F. N.; Análise Técnico-Econômica da Produção de Gasóleo Marinho (MGO) Sintético a partir de Roadmap. In: SIMPÓSIO DE PESQUISA OPERACIONAL E LOGÍSTICA DA MARINHA, 19., 2019, Rio de Janeiro, RJ. Anais [...]. Rio de Janeiro: Centro de Análises de Sistemas Navais, 2019.

\section{INTRODUÇÃO}

O gasóleo marinho (MGO) é uma das classificações do bunker, sendo produto da destilação atmosférica, ou seja, frações mais leves do processo de refino [1]. Seu preço está atrelado a alguns fatores, como o custo do refino de óleo cru, sua disponibilidade no local de abastecimento, as cotações internacionais, competitividade em relação aos principais portos localizados ao redor do mundo e custos de serviços relacionados ao abastecimento e transporte deste produto [2].

O mercado global deste combustível foi avaliado em 97 USD bilhões em 2016 e deverá atingir 147 USD bilhões até 2023. O aumento do comércio marítimo e, consequentemente, o aumento da frota marítima, é o fator que mais impulsiona este crescimento [3]. Porém novas estratégias para produção deste combustível com menor teor de contaminantes são necessárias, tendo em vista que em 2020 as regulamentações limitarão seu teor de enxofre em $0,5 \%{ }^{1}$ [4]. Nesta perspectiva, a utilização de combustíveis alternativos é bem vista no mercado. Dentro desta categoria destacam-se os sintéticos a partir da tecnologia Gas-to-Liquid (GTL) ${ }^{2}$. Em função da complexidade do processo Fischer-Tropsch, o uso de simulação é largamente empregado para sua avaliação técnica.

Além dos sintéticos, outros possíveis combustíveis podem ser empregados a uma perspectiva de curto e médio prazo. Para detectá-los, o uso de prospecção tecnológica visando a construção de um roadmap pode ser empregado. Portanto, a partir da análise técnicoeconômica e dos roadmaps, este artigo visa contribuir para a discussão sobre a utilização de combustíveis sintéticos no transporte marítimo bem como o mapeamento de novas tecnologias para produção/utilização de combustíveis alternativos. É esperado que este trabalho seja atrativo a empresas e centros de pesquisa que possuem interesse no setor de combustíveis marítimos, e que buscam por novas tecnologias para o novo segmento deste produto.

\section{METODOLOGIA}

\subsection{ELABORAÇÃO DO ROADMAP}

Estabeleceu-se o Google Patents como base de patentes e algumas estratégias de buscas, como o tipo da patente (depositada ou concedida) e o período de depósito/concedimento (2013 a 2018) utilizando as palavras-chave "("Alternative Fuel" OR "Alternative Bunker") AND ("Marine Transport" OR "Sea Transport") AND (Ships OR Vessels) AND Technology". A partir disto foram selecionadas 9 patentes concedidas (total de 13) e 17 solicitadas (total de 22) que mais se adequavam ao tema em questão. Após esta seleção, esquematizou-se os dados no Microsoft Excel, visando análise em 3 etapas [5][6]:

- Análise Macro - Foram contempladas informações imediatas do documento, como o título, ano, autor e sua origem, e o perfil do depositante;

\footnotetext{
${ }^{1}$ Já existem regulamentações que limitam o teor de enxofre em $0,1 \%$ em determinadas áreas de controle (desde 2015).

${ }^{2}$ Este processo visa converter gás natural pela síntese de Fischer-Tropsch em hidrocarbonetos de cadeia longa.
} 


\section{SPOLM2019

- Análise Meso - Realizou a leitura do resumo do documento a fim de extrair suas principais informações e elaborou-se as taxonomias;

- Análise Micro - Foram extraídas informações ainda mais aprofundadas para compreensão das taxonomias definidas.

\subsection{ANÁLISE TÉCNICA}

Utilizou o Aspen Hysys V10®, distribuindo a simulação em três etapas: geração do gás de síntese, síntese de Fischer-Tropsch e acabamento dos produtos. Utilizou-se o pacote SRK (Redlich-Kwong-Soave) com os componentes: alcanos (C1 a n-C20 e n-C25), alcenos (1-C2 a 1-C20 e C25), $\mathrm{H}_{2}, \mathrm{O}_{2}, \mathrm{~N}_{2}, \mathrm{CO}, \mathrm{CO}_{2}$ e $\mathrm{H}_{2} \mathrm{O}$. Onde $\mathrm{C} 1-\mathrm{C} 2$ representam gases leves, $\mathrm{C} 3-$ C4 GLP, C5-C9 nafta-gasolina, C10-C20 MGO e C25 graxa. A corrente de entrada de gás natural é composta por metano $(95,5 \%)$, etano $(3,00 \%)$, propano $(0,50 \%)$, butano $(0,40 \%)$ e nitrogênio $(0,6 \%)$, com vazão de $8195 \mathrm{kmol} / \mathrm{h}, 40{ }^{\circ} \mathrm{C}$ e $3000 \mathrm{kPa}[7]$.

\subsubsection{Geração do gás de síntese}

A corrente "GAS NATURAL" é misturada as correntes de reciclo para alimentar um trocador de calor a fim de aumentar sua temperatura a $455^{\circ} \mathrm{C}$, conforme ilustra a Figura 2.1. Essa corrente alimenta o pré-reformador (caracterizado pelo reator de Gibbs) em conjunto com a corrente "VAPOR DAGUA" (661 ${ }^{\circ} \mathrm{C}, 3000 \mathrm{kPa}$ e $\left.6524 \mathrm{kmol} / \mathrm{h}\right)$. É esperado que ocorra as seguintes reações no pré-reformador [7].:

$$
\begin{aligned}
& \mathrm{CO}+\mathrm{H}_{2} \mathrm{O} \leftrightarrow \mathrm{CO}_{2}+\mathrm{H}_{2} \\
& \mathrm{CO}+3 \mathrm{H}_{2} \leftrightarrow \mathrm{CH}_{4}+\mathrm{H}_{2} \mathrm{O}
\end{aligned}
$$

Figura 2.1 - Geração do gás de síntese.

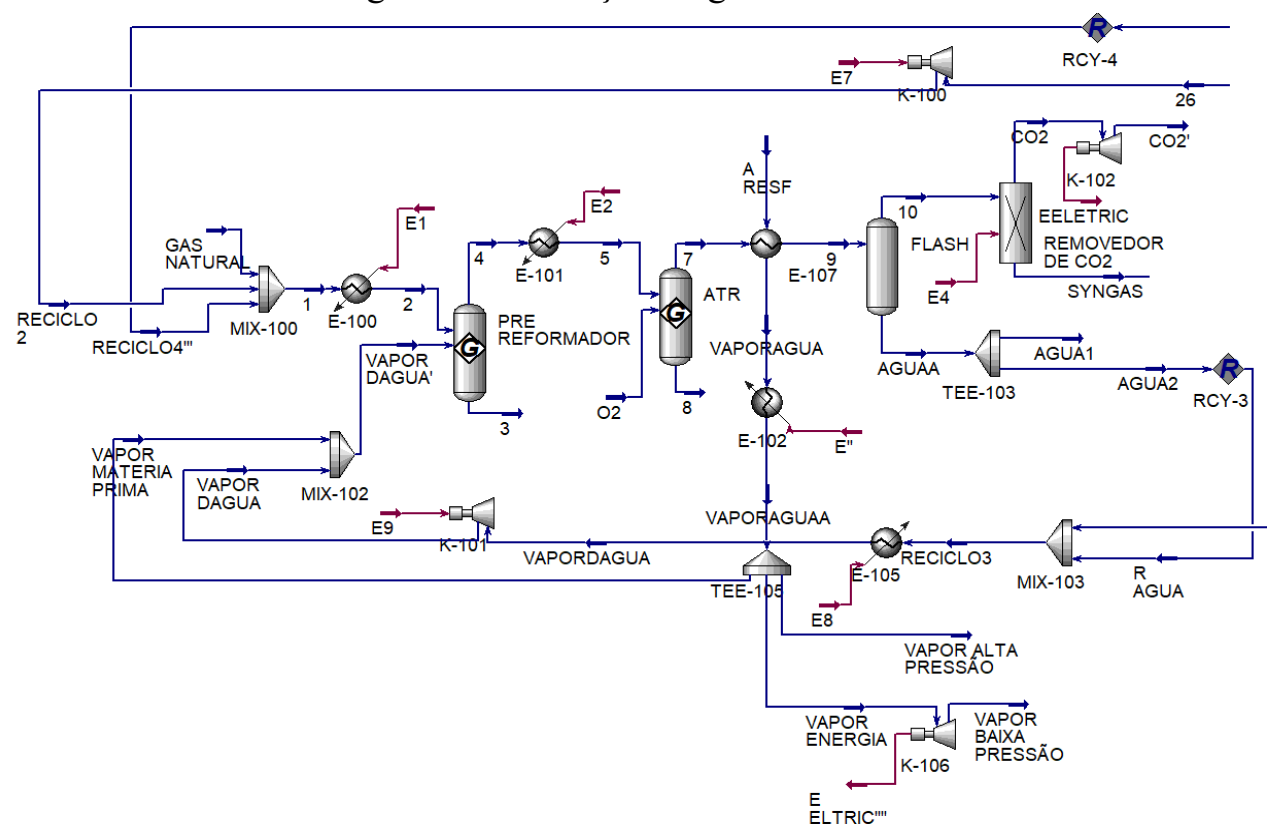

Fonte: elaborado pelo autor.

A corrente de topo do pré-reformador, alimenta o trocador de calor, para ser aquecida a $675^{\circ} \mathrm{C}$, resultando na corrente 5 , onde alimenta o reformador auto térmico (reator de Gibbs) em conjunto com a corrente "O 2 " $\left(200{ }^{\circ} \mathrm{C}, 3000 \mathrm{kPa}, 5236 \mathrm{kmol} / \mathrm{h}\right)$, considerando que ocorra as seguintes reações:

$$
\begin{aligned}
& \mathrm{CH}_{4}+3 / 2 \mathrm{O}_{2} \leftrightarrow \mathrm{CO}+2 \mathrm{H}_{2} \mathrm{O} \\
& \mathrm{CH}_{4}+\mathrm{H}_{2} \mathrm{O} \leftrightarrow \mathrm{CO}+3 \mathrm{H}_{2}
\end{aligned}
$$




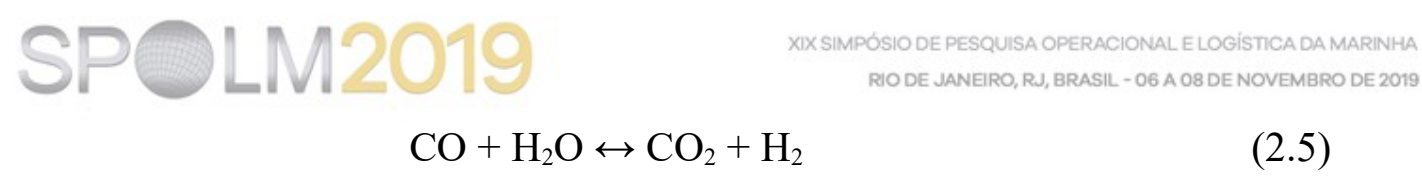

A corrente de topo é reduzida para $38^{\circ} \mathrm{C}$, onde alimenta um vaso de separação. Para esta troca térmica utilizou água de resfriamento $\left(10^{\circ} \mathrm{C}, 3000 \mathrm{kPa}\right.$ e $\left.48.887 \mathrm{kmol} / \mathrm{h}\right)$, onde é produzido vapor superaquecido para alimentar o pré-reformador. A corrente de topo segue para um removedor de $\mathrm{CO}_{2}$ (representado por um component splitter). A corrente de fundo é constituída pelo gás de síntese [7].

\subsubsection{Síntese de Fischer-Tropsch}

A corrente do gás de síntese passa por uma válvula, visando obter pressão de 2000 $\mathrm{kPa}$. Essa corrente é aquecida a $210^{\circ} \mathrm{C}$ para alimentar o reator, conforme ilustra a Figura 2.2.

Figura 2.2 - Síntese Fischer-Tropsch.

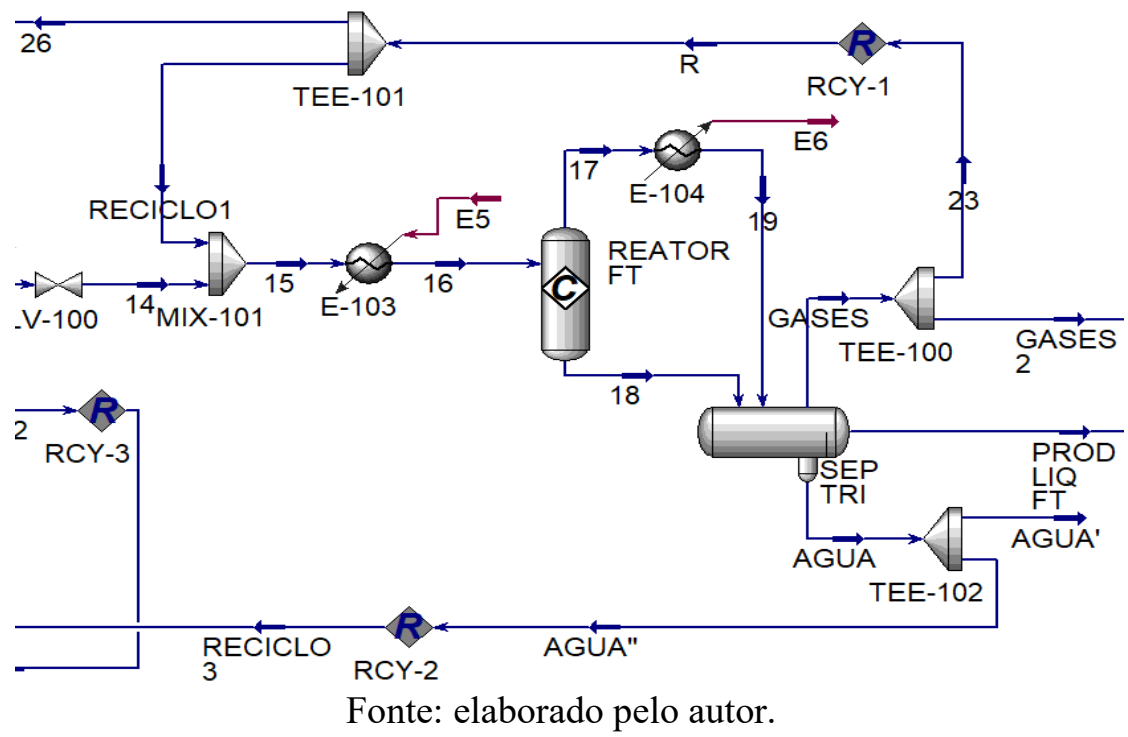

As reações de FT obedecem ao modelo de distribuição de Anderson-Schulz-Flory, de acordo com a equação 2.6, possibilitando calcular as frações mássicas (Wn) de cada produto através do $\alpha^{3}$ obtido pela equação 2.7. [7].

$$
\begin{gathered}
\mathrm{Wn}=\mathrm{n}(1-\alpha)^{2} \alpha^{\mathrm{n}-1} \\
\alpha=\left(0,2332\left(\mathrm{y}_{\mathrm{CO}} / \mathrm{y}_{\mathrm{CO}}+\mathrm{y}_{\mathrm{H} 2}\right)+0,633\right)[1-0,0039(\mathrm{~T}-533)]
\end{gathered}
$$

O valor de $\alpha$ calculado foi de aproximadamente 0,85 e a partir desse, calculou as frações mássicas e molares para cada componente. Foi adotado conversão de $70 \%$ para parafinas e $30 \%$ para olefinas. Do topo do reator, a corrente é resfriada a $35^{\circ} \mathrm{C}$ para alimentar um separador trifásico em conjunto com a corrente de fundo. Do separador, parte da corrente "ÁGUA" é reciclada para alimentar o pré-reformador. Outras duas correntes saem do mesmo separador, onde parte da corrente alimenta o "REATOR FT", parte é reciclada para alimentar o pré-reformador e parte alimenta um separador bifásico em conjunto com a corrente "PROD LIQ FT".

\subsubsection{Acabamento dos produtos}

\footnotetext{
${ }^{3}$ Probabilidade de crescimento da cadeia
} 
Essa separação é necessária para remover todas as frações gasosas presentes na corrente "PROD LIQ FT". Após a separação, parte da corrente de topo pode ser separada por splitters para remoção das correntes de GLP (Gás Liquefeito de Petróleo), propileno, etileno e etano. Parte da corrente 10\%) é purgada e o restante é recirculado para alimentar o préreformador, conforme ilustra as Figuras 2.1 e 2.3. A corrente líquida passa por uma válvula para que gere pressão atmosférica, onde alimenta uma torre de destilação. O produto de fundo dessa destilação também passa por uma válvula para que gere vácuo. Essa corrente então, alimenta outra coluna de destilação onde se separa MGO da graxa.

Figura 2.3 - Acabamento dos produtos.

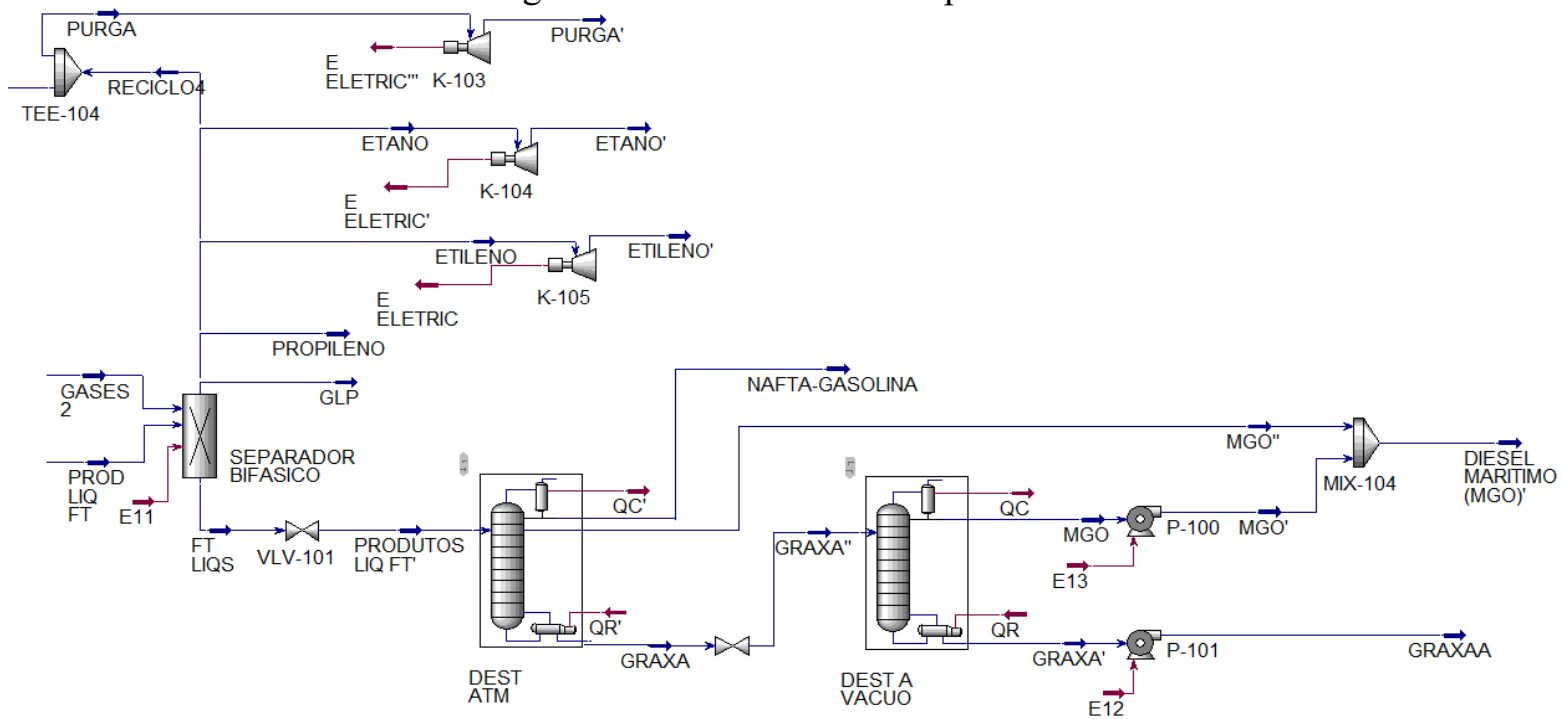

Fonte: elaborado pelo autor.

\subsection{ANÁLISE ECONÔMICA}

Esta análise se transcorreu em três etapas: estimativa dos custos de capital (Capital Expenditure - CAPEX), estimativa dos custos de operação (Operational Expenditure - OPEX) e cálculo do valor mínimo de venda do MGO. Para isto, foi necessário a definição de algumas premissas, como área demandada, localização e capacidade da planta, número de funcionários etc.

\subsubsection{Justificativa para o investimento}

O investimento proposto por este trabalho pode ser justificado pelas elevadas movimentações portuárias que ocorrem no Brasil, avaliadas em 325 milhões de toneladas de mercadorias em 2018. Para isto, uma grande frota de embarcações (exportadoras e importadoras) é necessária para transportar essas cargas, demandando elevadas quantidades de MGO para abastecer as mesmas [8]. Para estimar esta demanda, foram adotados alguns graus de liberdade, como:

- Os motores das grandes embarcações (14 cilindros - de 4000 a 5000 containers) consomem cerca de 4,1 toneladas de MGO por hora de percurso, considerando uma velocidade de 22 knots $(\sim 40 \mathrm{~km} / \mathrm{h})[9]$;

- Considera-se que os navios de carga que operam no Brasil transportam cerca de 4500 containers por viagem, cerca de $30 \%$ da capacidade do maior navio de carga do mundo (TRANSPORTABRASIL, 2019);

- Cada container armazena cerca de 25 toneladas [10]; 
- Tempo de navegação estimado: América do Norte, Europa e Ásia para o Brasil 192 h; América do Sul para o Brasil - 96 h; outros continentes para o Brasil - média de 192 h; Brasil para Brasil (cabotagem) - média de 24 h [8];

- Considera-se que cerca de 60 \% das embarcações importadoras, abasteçam no Brasil antes de retornar ao continente de origem;

- Considera-se que cerca de $60 \%$ das embarcações exportadoras e $30 \%$ das importadoras (dentro dos $60 \%$ ), atraquem e abasteçam no porto de Santos;

- Considera-se que cerca de $60 \%$ das embarcações de cabotagem atraquem e abasteçam no porto de Santos.

A partir dos graus de liberdade estabelecidos, estimou-se a demanda anual de MGO para o porto de Santos, com base nos dados estatísticos da ANTAQ (2019), conforme mostra a Tabela 2.1.

Tabela 2.1- Demanda de MGO para o porto de Santos em 2019

\begin{tabular}{|c|c|c|c|c|c|c|}
\hline $\begin{array}{l}\text { Trajeto das } \\
\text { Embarcações }\end{array}$ & Frota & $\begin{array}{c}\text { Frota que } \\
\text { abastece no } \\
\text { Brasil }\end{array}$ & $\begin{array}{c}\text { Frota que } \\
\text { abastece no } \\
\text { porto de } \\
\text { Santos } \\
\end{array}$ & Percurso & $\begin{array}{l}\text { Tempo de } \\
\text { Percurso }\end{array}$ & $\begin{array}{l}\text { Consumo de } \\
\text { MGO por } \\
\text { Frota porto de } \\
\text { Santos }(\mathrm{t}) \\
\end{array}$ \\
\hline \multicolumn{7}{|l|}{ Longo Curso } \\
\hline & \multirow{3}{*}{1588} & \multirow{3}{*}{1588} & 305 & BR-(1),(2),(3) & $192 \mathrm{~h}$ & 234.240 \\
\hline \multirow[t]{2}{*}{ Exportação } & & & 620 & BR - (4) & $216 \mathrm{~h}$ & 535.680 \\
\hline & & & 29 & $\mathrm{BR}-(5)$ & $96 \mathrm{~h}$ & 11.136 \\
\hline \multirow{3}{*}{ Importação } & \multirow{3}{*}{410} & \multirow{3}{*}{246} & 47 & \multirow{3}{*}{$\begin{array}{c}(1),(2),(3)-\mathrm{BR} \\
(4)-\mathrm{BR} \\
(5)-\mathrm{BR}\end{array}$} & \multirow{3}{*}{$\begin{array}{l}192 \mathrm{~h} \\
216 \mathrm{~h} \\
96 \mathrm{~h}\end{array}$} & \multirow{3}{*}{$\begin{array}{c}36.096 \\
12.960 \\
5.376\end{array}$} \\
\hline & & & 15 & & & \\
\hline & & & 14 & & & \\
\hline $\begin{array}{l}\text { Total Longo } \\
\text { Curso }\end{array}$ & 1998 & & 1030 & & & 835.488 \\
\hline Cabotagem & 564 & 564 & 339 & $\mathrm{BR}-\mathrm{BR}$ & $24 \mathrm{~h}$ & 32.544 \\
\hline Total & 2562 & 2398 & 1369 & & & 868.032 \\
\hline
\end{tabular}

(1) América do Norte; (2) Europa; (3) Outros; (4) Ásia; (5) América do Sul Fonte: adaptado de [8].

Nota-se a partir da Tabela 2.1 que a demanda estimada de MGO para o porto de Santos é de aproximadamente 868 kt para o ano de 2019, principalmente para abastecer os navios que exportam mercadorias para a Ásia. Diante disto, a planta proposta por este trabalho foi dimensionada para atender cerca de $80 \%$ desta demanda. Este valor foi determinado pois acredita-se que seja possível produzir um MGO sintético com preço mais atrativo do que o MGO fóssil comercializado no porto de Santos, desta forma, possuindo a preferência dos consumidores. O combustível proposto também apresenta teor de contaminantes $\left(\mathrm{S}_{\mathrm{x}}\right.$ e $\left.\mathrm{NO}_{\mathrm{x}}\right)$ significativamente menor, sendo mais atrativo aos países da América do Norte, Europa e alguns países da Ásia, pois através deste, torna-se possível transitar nas áreas de controle de emissões.

\subsubsection{Definição das premissas}

Utilizou-se o Aspen Process Economic Analyzer V10® (APEA), para dimensionar e estimar os custos dos equipamentos. Os valores estimados são referentes a base de custos na América do Norte, no primeiro trimestre de 2016, onde foi aplicado um fator de localidade 
para planta no Brasil de $16 \%$. O processo foi dimensionado para atender cerca de $80 \%$ da demanda prevista do MGO comercializado no porto de Santos. Para isto, este trabalho adotou como referência o Panahi e colaboradores (2012) [7], que utilizou dados da planta de Oryx $\mathrm{GTL}^{4}$ no Qatar para produzir $34.000 \mathrm{bbl} / \mathrm{dia}$ de produtos líquidos, e adaptado pelo presente trabalho para produzir cerca de $38.880 \mathrm{bbl} / \mathrm{dia}$, onde o MGO sintético representa 17.700 bbl/dia (691kta), conforme mencionado anteriormente. Também foi assumindo que o processo estaria em funcionamento cerca de 8.000 horas durante o ano. Esta previsão é estimada para um processo que opera 24 horas por dia, durante $90 \%$ dos dias no ano. Considera-se uma planta de $300 \mathrm{~m} \times 150 \mathrm{~m}^{5}$ e 300 funcionários $^{6}$ operando e 3 turnos.

\subsubsection{Estimativa do CAPEX}

Para esta estimativa, o cálculo foi subdividido em algumas etapas, como limites internos de bateria (Inside Battery Limmits - ISBL), contingência de processo, limites de externos de bateria (Outside Battery Limits - OSBL) e contingência do projeto. O cálculo do ISBL compreendeu os custos de aquisição e instalação dos equipamentos, onde foram introduzidos custos com tubulação, instrumentação, construção, fiação elétrica, pintura e isolamento, além de custos com estruturas ao redor do equipamento para proteção e facilidade ao acesso. Equipamentos não previstos, embora não componha o valor do ISBL, é uma contingência e foi calculado como $10 \%$ da soma dos custos de ISBL e OSBL [11].

O cálculo do OSBL foi constituído pelos custos dos equipamentos necessários para o funcionamento da instalação, como tanques para junção e divisão de correntes, Fired Heater, torres de arrefecimento, bombas, compressores, válvulas, turbinas, armazenamento para produtos, subprodutos e matérias-primas além da aquisição da área para construção da planta [8]. A contingência do projeto foi incluída para cobrir os custos que possam surgir ao longo da evolução do projeto. Esta contempla possíveis flutuações dos preços de materiais e mãode-obra, especificações incorretas de equipamentos, inflação, atrasos e outros imprevistos. A contingência do projeto está atrelada a complexidade da planta e a maturidade da tecnológica do processo, e foi definida como $25 \%$ [12].

\subsubsection{Estimativa do OPEX}

Essa estimativa foi calculada a partir das equações para o cálculo dos custos totais. A partir disto definiu-se a equação 2.8 para o cálculo do OPEX em conjunto com valores de operação de determinados equipamentos buscados na literatura. Onde $\mathrm{C}_{\mathrm{OP}}$ representa os custos com mão de obra dos operadores, $\mathrm{C}_{\mathrm{UT}}$ utilidades, $\mathrm{C}_{\mathrm{MP}}$ matérias-primas $\mathrm{C}_{\mathrm{C}}$ representa valor obtido com venda de créditos [8]

OPEX $=0,6805$ CAPEX $+3,16 \mathrm{C}_{\mathrm{OP}}+1,42\left(\mathrm{C}_{\mathrm{UT}}+\mathrm{C}_{\mathrm{MP}}-\mathrm{C}_{\mathrm{C}}\right)+$ OPEX scrubber de $\mathrm{CO}_{2}+$ OPEX Splitters de etano-etileno e propileno

\subsection{5 valor mínimo de venda do MGO}

Foram utilizados os valores de custos variáveis, custos fixos e despesas gerais, onde foi adicionado uma taxa de retorno de investimento. Essa taxa foi definida para se obter um payback ao CAPEX em 10 anos, a uma taxa interna de retorno (TIR) de 18\% [13].

\footnotetext{
${ }^{4}$ Empresa especializada em produtos GTL - seus principais acionistas: Sasol, Qatar Petroleum (QP), North Field e Ras Laffan Industrial City (RLC) (ORYXGTL, 2019).

${ }^{5}$ Dimensões estimadas baseadas na planta de ORYX GTL - Qatar

${ }^{6}$ A Sasol possuí média de 900 funcionários por país (ORYXGTL, 2019). Considera-se que possuí três filiais em cada país
} 


\section{RESULTADOS}

\subsection{ROADMAP}

Nota-se que a curto prazo há tendência para o uso de MGO sintético e o diesel dessulfurizado (Ultra Low Sulphur Diesel - ULSD) fóssil, conforme ilustra a Figura 3.1. Este fato pode estar atrelado a semelhança que estes combustíveis apresentam em relação aos utilizados atualmente, não necessitando de inovações nos motores das embarcações. Porém este cenário não se repete a médio prazo, onde o MGO sintético representa apenas $6 \%$ das patentes analisadas. A tendência deste cenário possivelmente está atrelada a complexidade do processo Fischer-Tropsch e suas elevadas condições de operação, podendo inviabilizar este produto comercialmente. A partir disto, tornam-se necessárias alternativas para sua produção visando flexibilizar as condições de operação.

Figura 3.1 - Cenário de curto, médio e longo prazo.

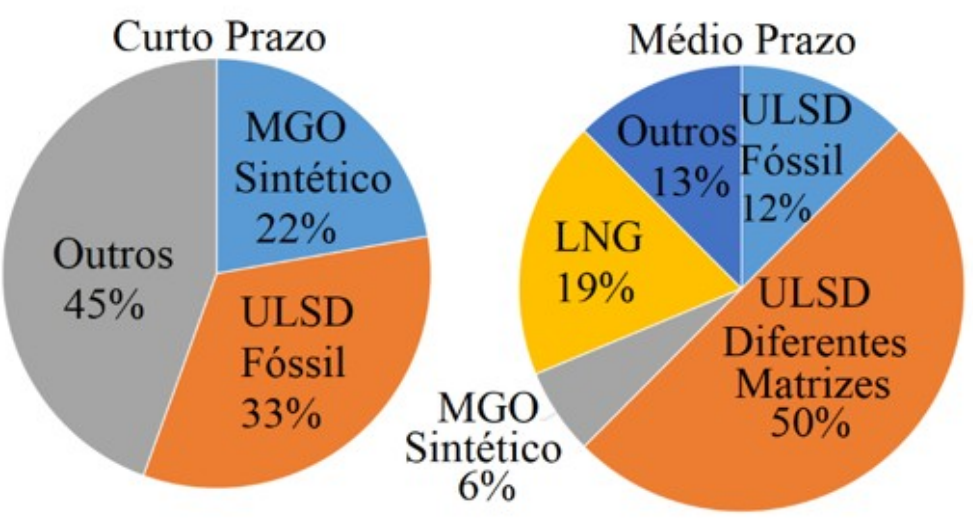

Fonte: elaborado pelo autor.

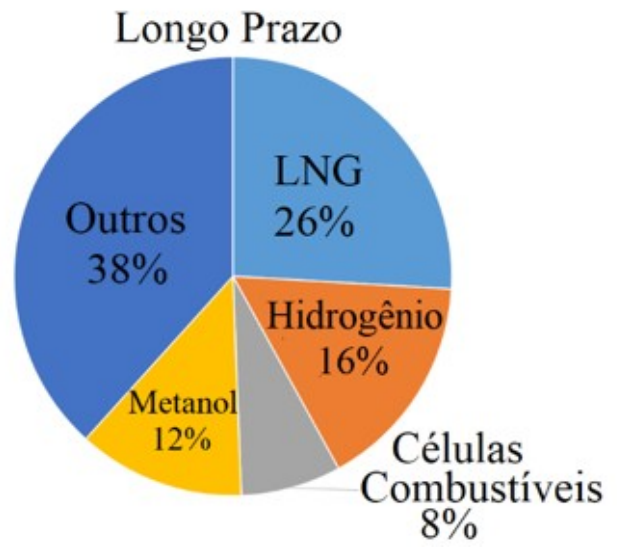

Diante disto, análise tecnico-econômica proposta por este trabalho é empregada para tal, com objetivo de reduzir o valor mínimo de venda do MGO sintético, tornando-o viável a curto e médio prazo. Este combustível possuí vantagem em relação ao ULSD (fóssil ou produzido por diferentes matérias-primas) pois apresenta teor de enxofre e nitrogênio significativamente inferior, atendendo as regulamentações de curto e médio prazo. Diferentemente do ULSD, pois como mencionado no capítulo de fundamentação teórica, é esperado que este combustível seja útil até 2021, quando as emissões de nitrogênio deverão apresentar queda de $85 \%$ [14].

Entretanto o MGO Sintético não atende as regulamentações de longo prazo quanto às regulamentações mais restritivas para emissões de $\mathrm{CO}_{2}$. Porém, pode ser utilizado a curto e médio prazo (cerca de 13 anos) para sedimentar a transição para os combustíveis gasosos como gás natural liquefeito (Liquefied Natural Gas - LNG) e hidrogênio a longo prazo. Dentro desta perspectiva, a planta proposta por este trabalho pode ser ajustada para produzir (em maiores frações) combustíveis que se enquadram nesta categoria, como por exemplo, o GLP. Assim efetivando um maior ciclo de vida (20 anos) para o projeto, uma vez que a planta produzirá combustíveis que atendem as regulamentações de longo prazo.

\subsection{ANÁLISE TÉCNICA}

Para a realização da análise técnica do processo proposto, foi necessário o estudo sobre algumas variáveis que influenciam na conversão do produto de interesse. Este estudo foi realizado com auxílio do Aspen Hysys, com objetivo de verificar a influência da temperatura do reator bem como a avaliação do uso de utilidades na planta e seu aproveitamento energético. 


\subsubsection{Influência da temperatura do reator nos produtos obtidos}

Para esta verificação, visando obter a maior conversão a MGO, elaborou-se a Figura 3.2 variando a temperatura do reator de 423 a $803 \mathrm{~K}^{7}$ em função das frações mássicas dos produtos obtidos. Esta análise é de suma importância para definir as condições operacionais do reator que fornecem maiores rendimentos para o produto desejado. Pode-se notar a partir da Figura 3.2 que a temperatura onde há maior conversão a MGO é $483 \mathrm{~K}\left(210{ }^{\circ} \mathrm{C}\right)$. De acordo com SASOL (2015) [15], essa temperatura é classificada como baixa temperatura de Fischer-Tropsch (Low Temperature Fischer Tropsch - LTFT). As condições reacionais de LTFT favorecem as frações mais pesadas dos produtos Fischer-Tropsch em função da temperatura de degradação das cadeias hidrocarbônicas, ou seja, quanto maior a temperatura reacional, mais leves serão as frações produzidas.

Figura 3.2 - Influência da temperatura do reator nos produtos obtidos. Influência da Temperatura nos Produtos

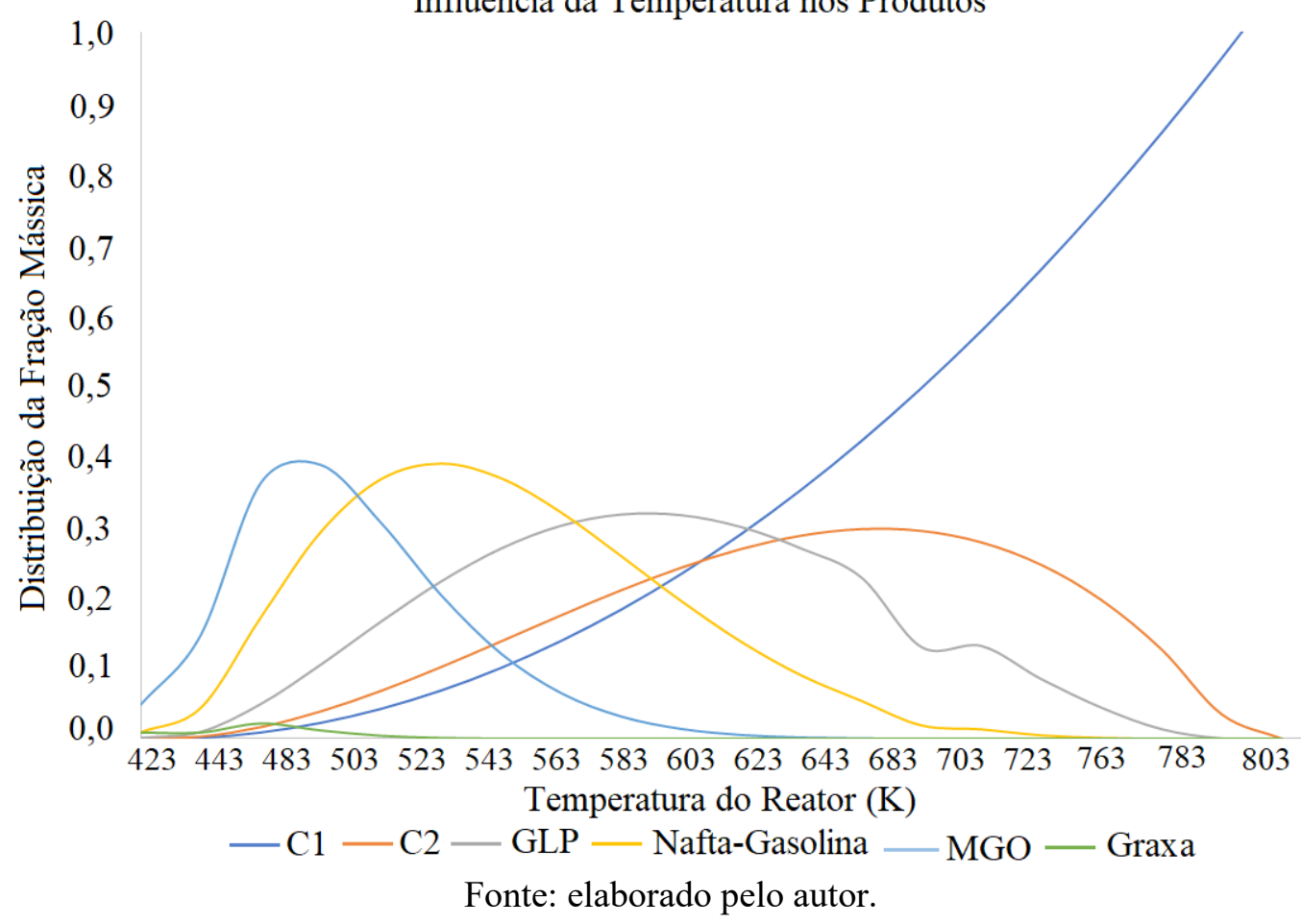

Nota-se também que em $803 \mathrm{~K}\left(530^{\circ} \mathrm{C}\right)$ há formação apenas de metano. Este fato não é interessante para as indústrias que compõe este setor, pois além deste produto possuir baixo valor agregado, é o mesmo utilizado na matéria-prima, ou seja, não haveria transformação química neste processo. Diante disso, um grande desafio é controlar a temperatura do meio reacional em função das reações serem extremamente exotérmicas, o que evidencia o elevado consumo de utilidades no processo.

\subsubsection{Consumo de utilidades no processo}

A demanda de utilidades para este processo engloba consumo de água de resfriamento, vapor de baixa (VBP) e alta pressão (VAP) e eletricidade. A Tabela 3.1 mostra as utilidades empregadas para cada equipamento utilizado na simulação, bem como as condições do processo e a carga térmica requerida por cada equipamento.

Tabela 3.1 - Demanda de utilidades para cada equipamento bem como sua carga térmica.

Utilidades Condições de Processo $\quad$ Equipamento Carga Térmica $(\mathrm{kW})$

\footnotetext{
${ }^{7}$ Intervalo de temperatura onde a Equação 2.7 é válida.

Equação 2.7: $\alpha=\left(0,2332\left(\mathrm{y}_{\mathrm{CO}} / \mathrm{y}_{\mathrm{CO}}+\mathrm{y}_{\mathrm{H} 2}\right)+0,633\right)[1-0,0039(\mathrm{~T}-533)]$
} 
SPOLM:

Vapor de Alta
Pressão (VAP)

Vapor de Baixa

Pressão (VBP)

Água de Resfriamento

$\mathrm{T}_{0}=40{ }^{\circ} \mathrm{C} \quad \mathrm{Tf}=455^{\circ} \mathrm{C}$

$\mathrm{T}_{0}=485^{\circ} \mathrm{C} \quad \mathrm{Tf}=675^{\circ} \mathrm{C}$

$\mathrm{T}_{0}=38^{\circ} \mathrm{C} \quad \mathrm{Tf}=455^{\circ} \mathrm{C}$

$\mathrm{T}_{0}=37^{\circ} \mathrm{C} \quad \mathrm{Tf}=210^{\circ} \mathrm{C}$

$\mathrm{T}_{0}=1226^{\circ} \mathrm{C} \quad \mathrm{Tf}=38^{\circ} \mathrm{C}$

$\mathrm{T}_{0}=210^{\circ} \mathrm{C} \quad \mathrm{Tf}=35^{\circ} \mathrm{C}$
XIX SIMPÓSIO DE PESQUISA OPERACIONAL ELOGISTICA DA MARINHA

RIO DE JANEIRO, RJ, BRASIL - 06 A O8DE NOVEMBRO DE 2019

$\begin{array}{cc}\text { E-100 } & 50.300,00 \\ \text { E-101 } & 50.506,00 \\ \text { E-105 } & 37.750,00 \\ \text { E-103 } & 26.860,00 \\ \text { E-107 } & -480.833,00 \\ \text { E-104 } & -81.444,00 \\ \text { K-100 } & 480,8 \\ \text { K-101 } & 1.527,00 \\ \text { P-100 } & 9,04 \\ \text { P-101 } & 2,3 \\ \text { Demais Bombas (20) } & 200,00 \\ \text { Uso em Geral } & 20.000,00\end{array}$

Fonte: elaborado pelo autor.

Como observado na Tabela 3.1, são elevadas as condições operacionais do processo Fischer-Tropsch, o que demanda elevadas quantias de utilidades, devido a carga térmica requerida para cada equipamento. Entretanto, este fato não se repete quanto ao uso de energia elétrica, pois, por mais que haja um elevado consumo, a planta é autossuficiente quanto ao uso dessa energia, devido ao uso de turbinas, conforme mostra a Tabela 3.2.

Tabela 3.2 - Energia elétrica produzida em relação ao consumo.

\begin{tabular}{cc} 
Equipamento & Carga Térmica $(\mathrm{kW})$ \\
\hline $\mathrm{K}-102$ & 324,80 \\
$\mathrm{~K}-103$ & 16,87 \\
$\mathrm{~K}-104$ & 125,10 \\
$\mathrm{~K}-105$ & 11,05 \\
$\mathrm{~K}-106$ & $70.430,00$ \\
Total & $70.907,82$ \\
Consumo & $22.219,14$ \\
Excesso & $48.688,68$ \\
\hline
\end{tabular}

Fonte: elaborado pelo autor.

Havendo ainda um excesso de energia produzida disponível, podendo ser comercializada como créditos, reduzindo o valor do MGO produzido. Diante da grande demanda de utilidades citada acima, integrações energéticas podem contribuir para redução deste consumo. Por exemplo, a água de resfriamento utilizada no trocador E-107 que passa por um fired heater, formando vapor de alta (VAP) e de baixa pressão (VBP), podem ser utilizadas na planta de forma que apresente redução de 448\% na demanda de VBP e $171 \%$ na demanda de VAP, conforme ilustra a Tabela 3.3. 
Tabela 3.3 - Economia no consumo de utilidades para aquecimento.

\begin{tabular}{lcccc}
\hline \multicolumn{1}{c}{ Utilidades } & Equipamento & $\begin{array}{c}\text { Vazão Mássica de Utilidade } \\
\text { Requerida (t/h) }\end{array}$ & Economia (t/h) & $\%$ \\
\hline Vapor de Alta & E-100 & 123,24 & 487,03 & $\mathbf{1 7 1 \%}$ \\
Pressão (VAP) & E-101 & 106,32 & & \\
Total & E-105 & 55,950 & & \\
Vapor de Baixa & E-103 & 285,51 & 305,60 & $\mathbf{4 4 8 \%}$ \\
Pressão (VBP) & 68,200 &
\end{tabular}

Fonte: elaborado pelo autor.

A Tabela 3.3 mostra que há um excesso de VAP e VBP produzido de cerca de $71 \% \mathrm{e}$ $348 \%$ respectivamente, podendo utilizar, também, nos reboilers pertencentes ao processo de destilação, conforme representado na Tabela 3.4.

Tabela 3.4 - Utilização de VBP produzido nos reboilers de destilação.

\begin{tabular}{|c|c|c|c|c|}
\hline Utilidade & Equipamento & $\begin{array}{l}\text { Vazão Mássica de } \\
\text { Utilidade Requerida } \\
(\mathrm{t} / \mathrm{h})\end{array}$ & Economia $(\mathrm{t} / \mathrm{h})$ & $\%$ \\
\hline Vapor de Baixa & Reboiler Dest Atm & 60,22 & \multirow{3}{*}{237,4} & \multirow{3}{*}{$382 \%$} \\
\hline Pressão & Reboiler Dest a Vácuo & 1,840 & & \\
\hline Total & & 62,06 & & \\
\hline
\end{tabular}

Fonte: elaborado pelo autor.

Foi escolhido utilizar VBP nesta etapa, pois o VAP possui maior valor agregado na venda como subproduto. De acordo com a Tabela 3.4, mesmo após esta operação, nota-se que ainda assim, há um excesso de $282 \%$ de vapor de baixa pressão produzido, evidenciando autossuficiência quanto a utilidades destinadas para o aquecimento, conforme ilustra a Figura 3.3. Este excesso, também pode ser comercializado como créditos para gerar economia nos custos do processo.

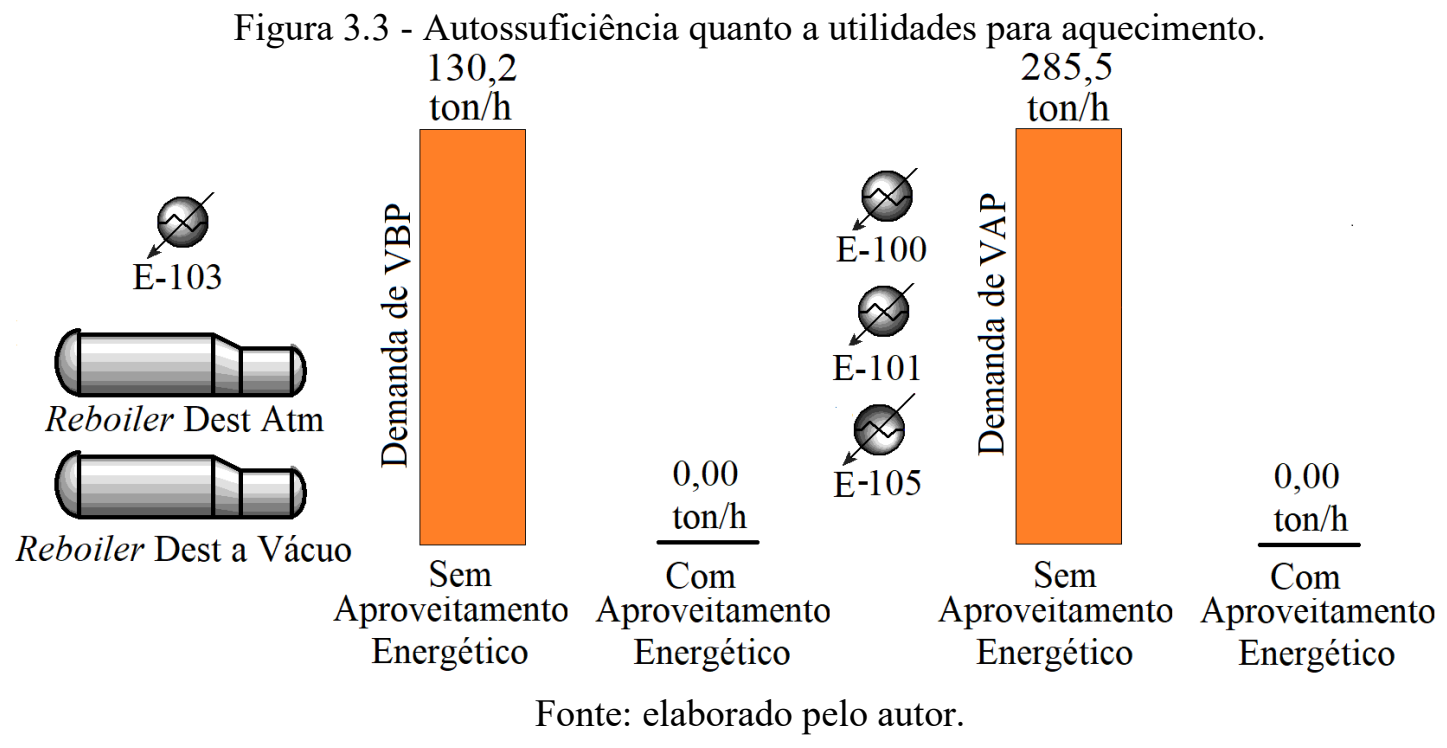

\subsection{ANÁLISE ECONÔMICA}

\subsubsection{Estimativa do CAPEX}

A Tabela 3.5 ilustra os valores do ISBL e OSBL em conjunto com as contingências de processo e projeto além de um ajuste inflacionário acumulado de $6,25 \%$. 


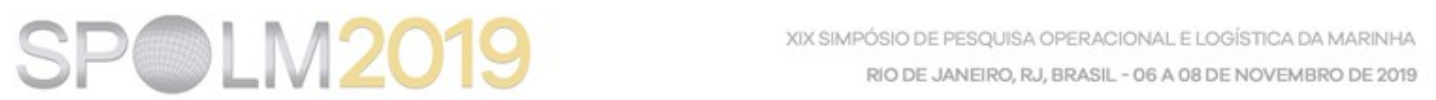

Tabela 3.5 - Estimativa do CAPEX

\begin{tabular}{lcc}
\hline Componente & Custo (USD Milhões) & \% CAPEX \\
\hline ISBL & 101,40 & $30 \%$ \\
OSBL & 109,04 & $32 \%$ \\
Contingência do Processo (10\% ISBL + OSBL) & 21,044 & $6 \%$ \\
Capital Total do Processo (CTP) & 231,48 & \\
Contingência do Projeto (25\% do CTP) & 57,871 & $17 \%$ \\
CAPEX (USA) 1 $1^{\circ}$ Trimestre de 2016 & 289,35 & \\
CAPEX (USA) 1 $1^{\circ}$ Trimestre de 2019 & 307,42 & \\
CAPEX (Brasil) & $\mathbf{3 5 6 , 6 1}$ & \\
\hline
\end{tabular}

Fonte: elaborado pelo autor.

Observa-se através da Tabela 3.5 que os custos com OSBL representam maior impacto sobre o CAPEX, devido aos elevados custos com tanques para armazenamento. Os custos com ISBL também possuem significante impacto, associado principalmente as etapas de acabamento dos produtos e geração de gás de síntese. Observa-se também que além das contingências, é acrescentado um valor de inflação acumulada de 6,25\% para atualizar o valor do CAPEX [16]. Também é adicionado um fator de localidade para o Brasil, de 16\% [17]. A partir disto, é obtido um custo de capital total de aproximadamente USD 356,61 milhões para uma planta a ser instalada no Brasil.

\subsubsection{Estimativa do OPEX}

Obteve-se um custo total de operação de cerca de 289,2 USD milhões, dos quais é constituído por $55 \%$ dos custos diretos totais, $23 \%$ dos custos fixos totais e $22 \%$ de despesas gerais, conforme ilustra a Tabela 3.6. Dentro dos custos diretos, destaca-se o elevado custo com matéria prima e o elevado custo de operação do splitter de propileno, porém estes valores são compensados devido ao elevado valor adquirido com a venda de créditos na planta, esta economia afeta significativamente o valor mínimo de venda do MGO produzido.

Tabela 3.6 - Estimativa do OPEX

\begin{tabular}{lc}
\hline \multicolumn{1}{c}{ Fator } & Custos (USD Milhões) \\
\hline Custos Diretos Totais & 160,6 \\
Custos Fixos Totais & 65,35 \\
Total Despesas Gerais & 63,22 \\
Custos Totais & $\mathbf{2 8 9 , 2}$ \\
\hline
\end{tabular}

Fonte: elaborado pelo autor.

\subsubsection{Valor mínimo de venda do MGO}

A Figura 3.4 ilustra as parcelas que compõe o valor mínimo de venda do MGO. Notase que se obteve um valor mínimo de venda de $547,25 \mathrm{USD} / \mathrm{t}$, dos quais é composto por $43 \%$ dos custos diretos, $36 \%$ dos custos fixos mais despesas gerais e $21 \%$ do retorno ao investimento. Este é um baixo valor em comparação com os disponíveis no mercado, pois considerou-se um cenário otimista onde se comercializa $100 \%$ de todos os subprodutos gerados como créditos, embora isto nem sempre aconteça. Para melhor compreensão sobre a flutuação do preço do MGO em relação a venda de créditos elaborou-se a Tabela 3.7 com os possíveis cenários de mercado. 


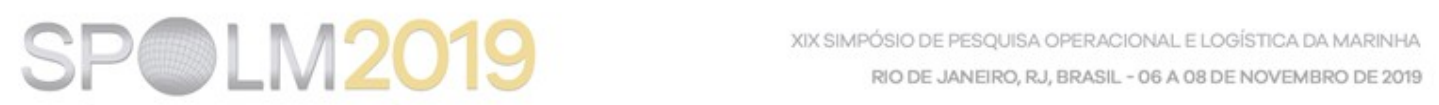

Figura 3.4 - Valor mínimo de venda do MGO.
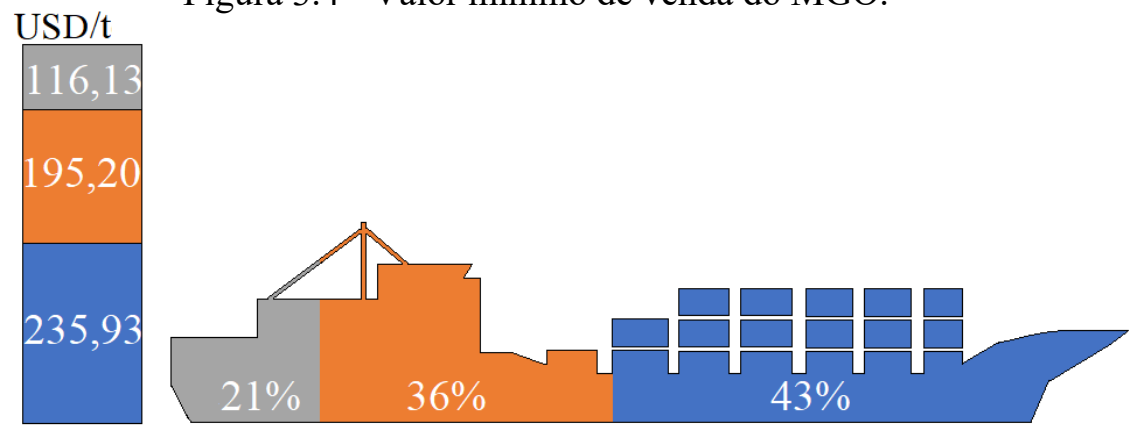

Custos Diretos

$\square$ Custos Fixos + Despesas Gerais

$\square$ Retorno do Investimento

Fonte: elaborado pelo autor.

Tabela 3.7 - Flutuações do preço do MGO com o cenário da venda de créditos.

\begin{tabular}{ccc}
\hline \multirow{2}{*}{ Cenário de Mercado } & $\begin{array}{c}\text { Valor Mínimo de Venda MGO } \\
(\text { USD/t) }\end{array}$ & Comercialização dos Créditos \\
\hline Otimista & 547,25 & $100,0 \%$ \\
Realista & 754,98 & $60,00 \%$ \\
Pessimista & 962,71 & $20,00 \%$ \\
\hline
\end{tabular}

Fonte: elaborado pelo autor.

Dentro deste cenário o GLP apresenta grande relevância, pois o preço deste subproduto compõe a grande maioria dos créditos comercializados $(65 \%)$. A partir disto é possível inferir que, quanto maior o preço deste subproduto, menor será o valor mínimo de venda do MGO. Porém este deve se manter em um preço atraente aos consumidores, para que maiores frações sejam comercializadas, atingindo proximidade ao cenário otimista, tornando o preço do MGO sintético competitivo com os demais. As Tabelas 3.8 e 3.9 ilustram as flutuações dos preços do MGO fóssil e ULSD nos principais portos do mundo em comparação com o MGO sintético proposto por esse trabalho.

Tabela 3.8 - Cenário de mercado do ULSD em comparação com o MGO sintético Flutuação dos Preços (USD/t)

\begin{tabular}{ccccccc}
\hline $\begin{array}{c}\text { Teor de } \\
\text { Enxofre }\end{array}$ & Combustível & Porto & $\begin{array}{c}\text { Baixo } \\
(2016)\end{array}$ & Médio & $\begin{array}{c}\text { Alto } \\
(2018)\end{array}$ & Referência \\
\hline \multirow{3}{*}{$0,1 \%$} & ULSD & Rotterdam & 352,00 & 518,50 & 712,00 & {$[18]$} \\
& ULSD & Singapura & 380,50 & 548,50 & 759,00 & {$[18]$} \\
& ULSD & Gibraltar & 392,25 & 569,00 & 763,75 & {$[18]$} \\
$\mathbf{0 , 0 0 \%}$ & MLSD & Piraeus & 379,50 & 561,00 & 758,75 & {$[18]$} \\
\hline
\end{tabular}

Fonte: elaborado pelo autor.

Tabela 3.9 - Cenário de mercado do MGO fóssil em comparação com o sintético.

Flutuação dos Preços (USD/t)

\begin{tabular}{ccccccc}
\hline $\begin{array}{c}\text { Teor de } \\
\text { Enxofre }\end{array}$ & Combustível & Porto & $\begin{array}{c}\text { Baixo } \\
(2016)\end{array}$ & Médio & $\begin{array}{c}\text { Alto } \\
(2018)\end{array}$ & Referência \\
\hline \multirow{2}{*}{$1,5 \%$} & MGO fóssil & Rotterdam & 353,75 & 519,50 & 711,00 & {$[19]$} \\
& MGO fóssil & Singapura & 378,50 & 544,50 & 756,25 & {$[19]$} \\
$0,5 \%$ & MGO fóssil & Piraeus & 383,50 & 560,00 & 767,00 & {$[19]$} \\
$\mathbf{0 , 0} \%$ & MGO fóssil & Santos & 578,50 & 701,00 & 854,75 & {$[19]$} \\
\hline
\end{tabular}

Fonte: elaborado pelo autor. 


\section{CONCLUSÃO}

A partir das análises referentes ao roadmap, pode-se inferir que, a curto prazo há forte tendência para o uso do MGO sintético e ULSD Fóssil, uma vez que são capazes de atender as regulamentações para transitar nas áreas de controle de emissões já em vigor. Estes também apresentam composição semelhante aos combustíveis utilizados atualmente, não impondo a necessidade de alteração nos motores das embarcações já existentes. O uso do ULSD fóssil pode auxiliar no cumprimento das regulamentações atuais, no entanto, não haveria redução na dependência dos combustíveis fósseis. A partir disto nota-se que há elevada tendência para produção deste por diferentes matérias-primas a médio prazo.

Esta produção pode ser uma alternativa, devido ao grau de sustentabilidade atribuída a este combustível, uma vez que se utilizam óleos descartados por diversos processos para sua produção. Entretanto, por mais que o ULSD fóssil ou produzido por diferentes matériasprimas, cumpra as especificações quanto ao teor de enxofre, não serão capazes de atender as regulamentações que passam a entrar em vigor a partir de 2021 em relação a queda de $85 \%$ nas emissões de NOx, conforme relata McVay (2015) [14]. Dentro deste cenário, surge o MGO sintético como grande ator, pois apresenta vantagem em relação ao ULSD, devido a sua composição de nitrogênio significativamente reduzida. Portanto, cumprindo as regulamentações. O que justifica a análise técnico-econômica para o MGO sintético, quanto a aquisição de uma planta cujo produto atenda as regulamentações de curto e de médio prazo.

Entretanto, ao analisar o roadmap de médio prazo, nota-se que há baixa tendência para o uso deste combustível, possivelmente devido às elevadas condições operacionais que demandam o processo de Fischer-Tropsch, inviabilizando o MGO sintético comercialmente. No entanto, dentro desta perspectiva, a análise técnico-econômica realizada por este trabalho contribuiu para discussão sobre a mudança deste cenário, uma vez que foi possível obter este combustível com preço competitivo (547 USD/t), ainda que hajam possíveis flutuações. Este preço está atrelado as integrações energéticas utilizadas no processo, bem como a comercialização de subprodutos como créditos. Tais eventos atribuíram autossuficiência à planta proposta por este trabalho, uma vez que as demandas por água de resfriamento, oxigênio e gás natural podem ser contornadas ao instalar esta planta na vizinhança de indústrias que possam fornecer estes insumos e utilidades.

Ao considerar um cenário otimista para o segundo trimestre de 2019, nota-se que MGO sintético proposto por este trabalho, apresenta preço mais baixo do que o fóssil comercializado no porto de Santos e nos demais portos citados, também apresentando preço atrativo em comparação ao ULSD. Seu preço se mantem competitivo mesmo ao considerar um cenário realista, entretanto, isto não ocorre para o cenário pessimista. Evidenciando que o valor dos créditos deve variar apenas de $60 \%$ a $100 \%$ para que o MGO sintético seja atrativo para indústria marítima.

Em vista dos cenários apresentados, por mais que a proposta apresentada neste trabalho tenha se mostrado eficiente, possui vida útil de apenas 13 anos, em função do MGO sintético não atender às regulamentações mais restritivas quanto as emissões de $\mathrm{CO}_{2}$ após esta data. Desta forma, é esperado que este combustível irá sedimentar a transição para os combustíveis gasosos, como o LNG, hidrogênio, conforme observado no roadmap de longo prazo. Por outro lado, o processo pode ser ajustado para produzir esta categoria de combustível, como por exemplo, o GLP. Desta forma, resultando em um projeto com maior ciclo de vida, cerca de 20 anos, atendendo às regulamentações de curto, médio e longo prazo, evidenciando a flexibilidade e eficiência da planta proposta. 


\section{S50142019}

\section{REFERÊNCIAS BIBLIOGRÁFICAS}

[1] FARAH, M. A. Petróleo e seus derivados. LTC, 2012.

[2] DRAFFIN, N. Commercial practice in bunkering. Oxfordshire: Petrospot, 2011.

[3] BUNKER FUEL - GLOBAL MARKET OUTLOOK (2017-2023). Disponível em: https://www.researchandmarkets.com/reports/4426140/bunker-fuel-global-market-outlook2017-2023. Acesso em: 19/09/2018

[4] DRAFFIN, N. An introduction to bunkering. 2nd ed. Oxfordshire: Petrospot, 2012.

[5] BORSCHIVER, S.; SILVA, A. TECHNOLOGY ROADMAP - Planejamento Estratégico para alinhar Mercado-Produto-Tecnologia. Ed. Interciência, 2016.

[6] COUTINHO, Paulo; BOMTEMPO, José Vitor. ROADMAP TECNOLÓGICO EM MATÉRIAS-PRIMAS RENOVÁVEIS: UMA BASE PARA A CONSTRUÇÃO DE POLÍTICAS E ESTRATÉGIAS NO BRASIL. Química Nova, São Paulo, v. 34, n. 5, p.1-13, 2011.

[7] PANAHI, M. et al. A Natural Gas to Liquids Process Model for Optimal Operation. Industrial \& Engineering Chemistry Research, [s.1.], v. 51, no 1, p. 425-433, 2012. ISSN: 0888-5885, DOI: 10.1021/ie2014058.

[8] ANTAQ. Agência Nacional de Transportes Aquaviários - Estatísticas. Disponível em: $<$ http://web.antaq.gov.br/Anuario/>, acesso em junho de 2019.

[9] TRANSPORTGEOGRAPHY. Fuel Consumption by Containership Size and Speed. Disponível em: <https://transportgeography.org/?page_id=5955>, acesso em junho de 2019.

[10] TRANSPORTABRASIL. CONHEÇA O MAIOR NAVIO CARGUEIRO DO MUNDO. Disponível em: < HTTP://WWW.TRANSPORTABRASIL.COM.BR/2012/09/CONHECAO-MAIOR-NAVIO-CARGUEIRO-DO-MUNDO/>, acesso em junho de 2019.

[11] TURTON, R. et al. Analysis, Synthesis, and Desing of Chemical Processes. New Jersey: Pearson Educarion, Inc., 2012.

[12] INTRATEC. Bidiesel Production from Soybean Oil- Cost Analysis- Biodiesel E11A. [S.1.]. 2017.

[13] ZHANG, Chundong et al. Efficient utilization of carbon dioxide in gas-to-liquids process: Process simulation and techno-economic analysis. Fuel, [s.1.], v. 157, p.285-291, out. 2015. Elsevier BV. http://dx.doi.org/10.1016/j.fuel.2015.04.051. 
[14] MCVAY J. (2015) Future Marine Fuel Quality Changes: How might terminals prepare?. Published by IHS Energy, Oil Markets and Downstream (OMDS) Consulting.

[15] SASOL TECHNOLOGY (PTY) LTD, JOHANNESBURG (ZA) (United States). United States Patent Office. Luis Pablo Fidel Dancuart Kohler; Paulus Stephanus Gravett; Jacques van Heerden. Heavy synthetic fuel. US nº US2015/0072298A1, 05 mar. 2012, 05 mar. 2013. 2015.

[16] INFLATION DATA. Disponível em: < https://inflationdata.com/Inflation>. Acesso em maio de 2019.

[17] INTRATEC. Plant Location Factors. Intratec, 2019. Disponivel em: $<$ https://www.intratec.us/indexes-and-pricing-data/location-factor/brazil $>$. Acesso em: 20 de abril de 2019.

[18] ROTTERDAM BUNKER PRICES. Disponível em: $<$ https://shipandbunker.com/prices/emea/nwe/nl-rtm rotterdam\#LSMGO $>$ Acessado em 30 de abril de 2019.

[19] SANTOS BUNKER PRICES. Disponível em: $<$ https://shipandbunker.com/prices/am/samatl/br-ssz-santos\#MGO $>$. Acesso em 30 de abril de 2019 